\section{Arte y educación emocional: una propuesta en la formación inicial de maestros}

\title{
Resumen
}

Son múltiples las investigaciones que ponen de manifiesto la pertinencia y necesidad de dotar al profesorado de una sólida formación en inteligencia emocional que les permita gestionar las emociones creadas en el grupo-clase, así como las suyas propias. A su vez, existe una necesidad por parte dicho profesorado de conocer técnicas y recursos para poder desarrollar esa inteligencia emocional en su alumnado.

Atendiendo a esta necesidad, el presente artículo muestra una propuesta desarrollada en el grado de maestro en Educación Primaria de la Universidad de Barcelona en el contexto de la asignatura de educación visual y plástica, con el objetivo de contemplar la educación emocional en la formación de los futuros docentes desde el área de expresión artística.

Con el objetivo de evaluar el impacto emocional de dicha experiencia en el alumnado, se proporcionó un cuestionario en el que se recogían datos cualitativos de aspectos vinculados a la misma.

Los resultados indican que la práctica desarrollada ha contribuido a que el alumnado participante incrementase su conocimiento sobre sus propias emociones y reflexionase sobre el papel de las mismas en la educación. Además constatamos, a partir de las propuestas de adaptación planteadas por el alumnado, que la batería de actividades realizada les ha dotado de herramientas para implementar en sus futuras aulas.

Palabras clave: Educación Visual y Plástica; Educación emocional; Formación del profesorado

\section{Emotional education and art: a plan for the initial teacher training}

\section{Abstract}

Multiple investigations have expressed the need of providing teachers with a solid formation in emotional management, as this will allow them to conduct not only the feelings created in the class or group, but also their own. At the same time, it is necessary for teachers to know the techniques through which they can develop the emotional intelligence of their pupils.

\footnotetext{
a Universitat de Barcelona

b Universitat de Barcelona

c Universitat de Barcelona

${ }^{d}$ Universidad Internacional de La Rioja
} 
In response to this need, this article shows a proposal developed in the course of plastic and visual education as a part of the bachelor in primary teacher at the University of Barcelona (UB). The aim of this study is to introduce emotional education as a part of the formation of future professors on the field of artistic expression.

The results show that our proposal has helped the students to increase the knowledge about their own emotions and, furthermore, to become more conscious about the role that emotions play in education. Moreover, the proposals applied in this study have also been seen to provide the students with tools which could be implemented in their future lectures.

Keywords: plastic and visual education; emotional education; initial teacher training.

\section{Introducción}

Hacia medidos de los años 80 , la visión tradicional de la inteligencia que ponía énfasis en los aspectos cognitivos entró en crisis por dos motivos: por una parte se cuestionó que la inteligencia académica fuese suficiente para alcanzar el éxito profesional y, por otra, porque lo que hasta entonces se entendía por inteligencia no era garantía de éxito en nuestro día a día (Molero et al. 1998). Como respuesta a este cuestionamiento crítico a las consideraciones que durante años el conductismo y el positivismo lógico habían hecho de las emociones (Bisquerra, 2000) así como de la visión tradicional de inteligencia, surgieron planteamientos que prestaban especial atención a los aspectos emocionales. Entre estos podemos destacar las teorías propuestas por Stermberg (1985), Mayer y Salovey (1993) o Goleman (1995).

Desde entonces las emociones han adquirido un nuevo papel cultural, lo que ha conllevado importantes cambios tanto psicológicos, como sociales y pedagógicos (Zaccagnini, 2008). En este contexto, parece necesario también articular mecanismos y programas para educar emocionalmente a la ciudadanía y, para ello, dotar a los maestros y estudiantes de maestro de herramientas que les permitan desarrollar las competencias socio-emocionales tanto del alumnado como las suyas propias. A pesar de esta necesidad, y tras analizar los planes de estudio del Grado de Maestro de Educación Primaria de 15 de las principales universidades españolas ${ }^{1}$, constatamos que en ninguno de ellos se contempla una asignatura de carácter obligatorio sobre educación emocional. Solo aparecen como optativas en la Universidad Autónoma de Madrid, donde encontramos la asignatura "Habilidades Socio-Emocionales para Alumnos con Problemas de Relación" en la Mención en Educación Inclusiva, y en la Universidad de Santiago de Compostela la asignatura optativa general "Educación Emocional".

A pesar de esto, el valor y potencialidad de la educación emocional en las aulas es una realidad observable a través de diversas acciones tales como el interés creciente y constante en el Postgrado y Master de Educación Emocional en la Universidad de Barcelona, que ya cuenta con 15 ediciones, así como las Jornadas y Congresos de Educación Emocional que se suceden a lo largo de la geografía española desde hace más de una década o la gran cantidad de formación complementaria a través de los llamados MOOC (Massive Online Open Courses) centrada en la temática. Tampoco hay que olvidar la creciente predilección del alumnado que a punto de finalizar el Grado de Maestro (Infantil o primaria) o de Educación Social desean centrar la temática de su trabajo Final de Grado alrededor del concepto de la Educación Emocional (desde propuestas de material, asignaturas, estudios de casos de escuelas que han implementado una metodología basada en la educación emocional...), viendo en ello una oportunidad de trabajar esta materia de una forma profunda y suplir las carencias que bajo su punto de vista el grado no le ha sabido ofrecer.

Así, conscientes de la necesidad de incorporar la educación emocional en el bagaje pedagógico del futuro profesorado, desde el área de expresión plástica se ideó una experiencia a modo de prueba piloto que se implementó en la facultad de educación de la Universidad de Barcelona enmarcada en el curso 2015/2016. Esta propuesta se presenta en este artículo. 


\section{Una aproximación a las emociones}

La relación entre las artes y las emociones es un tema ampliamente debatido, a pesar de lo cual la literatura científica se encuentra aún en un proceso de delimitación y concreción. Las diversas teorías que pretenden argumentar y analizar las emociones se agrupan en torno a diferentes perspectivas, tales como la evolucionista (Izard, 2007), la psicofisiológica (Souza, 2011), la neurológica (Palmero, 1996), la conductista (Fernández-Abascal, Chóliz, Palermo y Martínez, 2007) o la cognitiva (Mathews y MacLeod, 1994), y todas tienen como objetivo consolidar el conocimiento que se tiene sobre los procesos emocionales.

En la actualidad no existe una perspectiva predominante sino que se tiende a la combinación de todas ellas en pro de un estudio holístico de la emoción que integre diversas disciplinas como la psicología, la neurociencia, la pedagogía, etc. En cualquier caso, a lo que sí se ha llegado es a cierto consenso científico de carácter transversal e integrador respecto a entender la emoción como "un estado complejo del organismo caracterizado por una excitación o perturbación que predispone a una respuesta organizada" (Bisquerra, 2000: 61).

Las emociones se generan como respuesta a un acontecimiento externo o interno, de forma que cuando un sujeto percibe una situación o un estímulo realiza una primera valoración sobre si es positivo o negativo para la consecución de un objetivo determinado. De esta forma, se ve envuelto en una situación de placer o displacer-valencia- que refleja a través de una serie de mecanismos corporales tales como alteraciones en la respiración, en el tono muscular o en el ritmo cardiaco -arousal-. Estos dos factores, el placer o displacer y la activación o falta de ella que un estímulo nos produce, son independientes entre sí y permiten caracterizar las emociones (Russell, 1980).

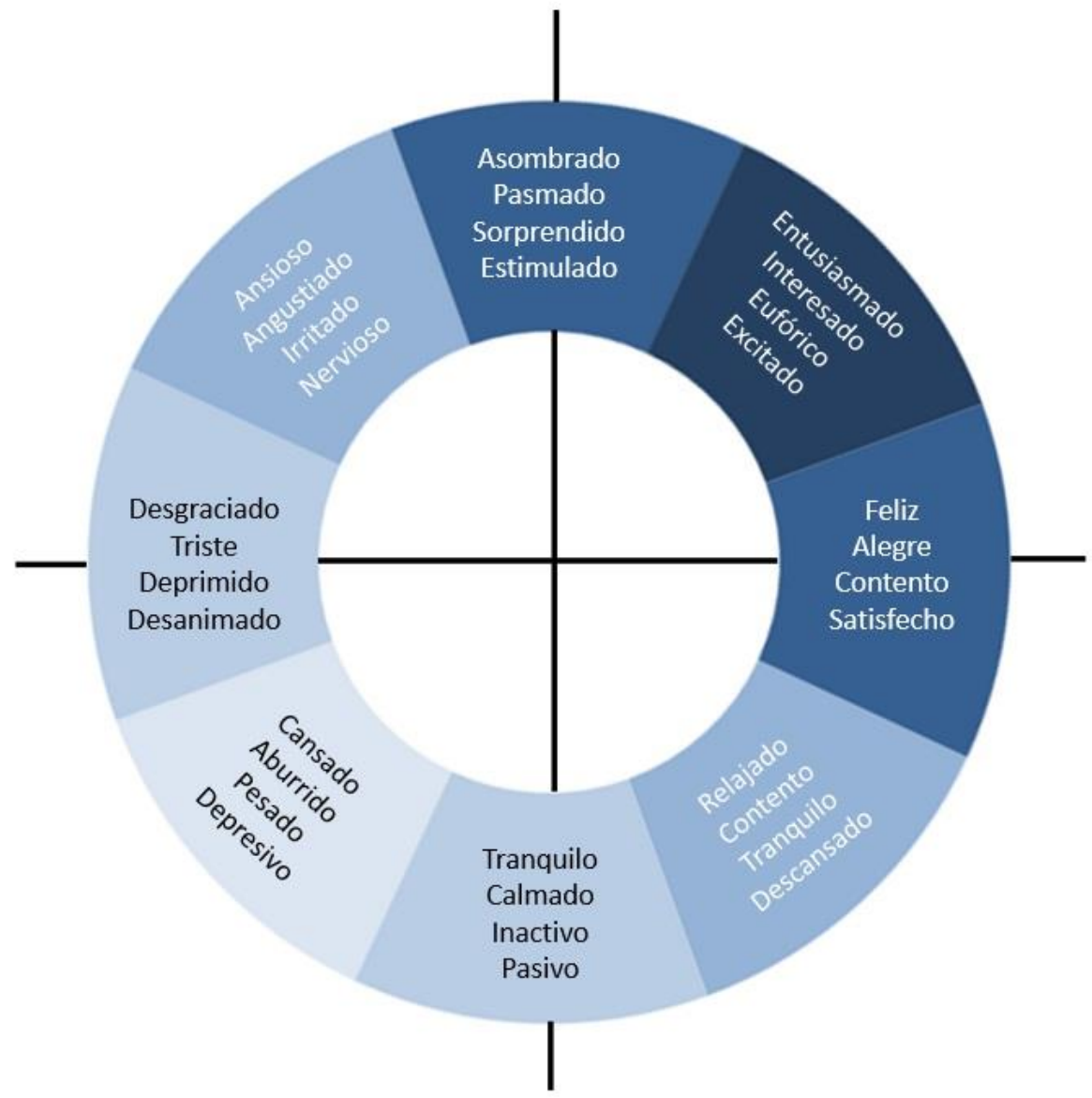

Figura 1. Modelo circunflejo de las emociones. Elaborado a partir de Rusell (1980) 
Por lo que respecta a la tipología de las emociones, existen tantas como modelos teóricos propuestos. Uno de los más extendidos es el que defiende la existencia de una serie de emociones básicas innatas y universales, a partir de las cuales se articulan el resto de reacciones afectivas o emociones secundarias (Izard, 2001). Otro modelo que cuenta con gran respaldo dentro de los ámbitos científicos es el que propone la agrupación de emociones en cuatro familias en función de su especificidad. De esta forma podríamos distinguir entre las emociones negativas, las emociones positivas, las emociones ambiguas y las emociones estéticas (Bisquerra, 2000).

De la conjugación de estas cuatro tipologías se desprenden lo que Bisquerra y Pérez (2007) denominan como las competencias emocionales: consciencia emocional -capacidad para tomar conciencia de las emociones propias y de las de los demás-; regulación emocional-capacidad para manejar las emociones de forma apropiada-; autonomía emocional -conjunto de características relacionadas con la autogestión personal tales como la autoestima, la actitud positiva, etc.-; competencia social -capacidad para mantener buenas relaciones con las otras personas-; y competencias para la vida y el bienestar capacidad para adoptar comportamientos apropiados y responsables para afrontar los desafíos diarios. El correcto desarrollo de todas ellas nos invita a pensar en una persona competente en los contextos laborales, personales, sociales, familiares, etc.

Desde la práctica artística, son numerosas las experiencias sobre terapias relacionadas con la mejora de los individuos que presentan problemas como alternativa a la psicoterapia tradicional o simplemente refuerzan canales de comunicación interpersonales e intrapersonales. En estas terapias el proceso consiste en la toma de conciencia que se produce a partir de las producciones en tercera persona (Klein, 2006). De esta forma, la posibilidad que tiene cualquier individuo de ser un artista, entrando en conversación consigo mismo y conectando con sus emociones más íntimas, se convierte en un proceso cercano a la catarsis (Riera, 2010). Proceso en el que se puede distinguir en función de la técnica artística que se emplee. Por ejemplo, la música como base de la Musicoterapia (Betes, 2000), las artes escénicas como sustento de la Dramaterapia (Cornejo y Brik, 2003), o los medios visuales y plásticos para experimentar la Arteterapia (Klein, 2006).

Se han realizado múltiples investigaciones que constatan los beneficios de cada una de estas técnicas terapéuticas, a pesar de lo cual son escasos los estudios sobre el arte como medio desde el enfoque de la psicología positivista, es decir, como modelo preventivo de la salud a partir de las fortalezas y resiliencia de los participantes, que actúe como medida profiláctica y de crecimiento personal ante enfermedades mentales a través del autoconocimiento y la autoestima (Gustems y Sánchez, 2015) o, simplemente, que refuerce la educación emocional de los sujetos hasta poder considerarlos inteligentes emocionalmente hablando (Goleman, 1999).

En ese refuerzo, y volviendo a la Arteterapia, el Siglo XXI nos está planteando retos que la expresión artística desde el área visual y plástica parece estar abordando con éxito (Callejón y Granados, 2003). Éxito basado en propuestas en las que el inconsciente puede olvidarse de las palabras razonadas y entrar en el terreno de la simbología y las imágenes (Duncan, 2007). Éxitos que se reflejan tanto en la infancia (Rodríguez, 2007) como en los difíciles años de la adolescencia (Oriola y Gustems, 2016), al igual que en los adultos (Villacreses, Callejas, Santamaría y Albornoz, 2014), e incluso propuestas que se dirigen a toda una comunidad (Burset, Calderón y Gustems, 2016).

\section{La necesaria alfabetización emocional del profesorado}

Los cambios pedagógicos a los que nos referíamos al inicio del presente artículo, guardan estrecha relación con el cuestionamiento de si la simple instrucción es suficiente para cumplir los objetivos de la educación obligatoria y las demandas de una realidad cambiante (Marina et al. 2015) lo que se ha venido a llamar "modernidad líquida" (Bauman, 2005). En este contexto se ha puesto en valor la enseñanza basada en competencias, impulsada por el informe de la UNESCO (Delors et al. 1996) que establecía los pilares básicos de la educación: «aprender a conocer», «aprender a hacer», «aprender a ser»y «aprender 
a convivir» entendiendo la necesidad de que el alumnado adquiera un rango de competencias que le permita disfrutar de una vida social y personal acorde con la sociedad actual.

Como desarrollo posterior de este marco conceptual, aparecen las acciones impulsadas desde el Espacio Europeo de Educación Superior (EEES), desarrolladas y concretizadas por la LOMCE, Ley Orgánica $8 / 2013$, de 9 de diciembre, establece las siguientes competencias clave ${ }^{2}$ para la educación primaria:

a) Comunicación lingüística.

b) Competencia matemática y competencias básicas en ciencia y tecnología.

c) Competencia digital.

d) Aprender a aprender.

e) Competencias sociales y cívicas.

f) Sentido de iniciativa y espíritu emprendedor.

g) Conciencia y expresiones culturales.

Con todo ello se busca el desarrollo integral del alumnado, en el que debería emerger la inteligencia emocional, aunque no se explicite como tal. Las competencias personales y sociales contempladas como e) y f) serían una aproximación a lo que podríamos denominar competencia o educación emocional. Además esto exige que el docente tenga desarrolladas sus competencias emocionales, ya que no es posible enseñar una competencia que no se ha alcanzado previamente, por lo que se hace evidente la necesidad de una formación del profesorado en este ámbito, ya sea inicial o continua (Cabello et al., 2009; Fernández-Berrocal y Ruiz-Aranda, 2008; Palomera et al., 2008). En esta línea, un estudio reciente (Cejudo et al., 2015) muestra que los futuros maestros son sensibles a esta necesidad de formación en lo que a educación emocional se refiere, pues son conscientes de las implicaciones y la importancia que las emociones desempeñarán en su futura labor.

\section{Objetivos y diseño de la propuesta}

En este marco contextual que acabamos de presentar, e incidiendo en la necesaria formación del profesorado, es donde se desarrolla la propuesta de intervención educativa que se presenta. Nuestro objetivo fue evaluar el impacto emocional percibido a través de una experiencia basada en herramientas de la didáctica de la expresión visual y plástica en el alumnado de grado de maestro en educación primaria.

Para ello, se creó una batería de propuestas las cuales se implementaron en el aula universitaria. De esta forma se buscó dotar al futuro profesorado de herramientas de educación emocional para trabajar en el área de expresión artística. Posteriormente se ha evaluado el impacto que esta implementación ha tenido, lo cual nos permite extraer conclusiones sobre la idoneidad de la experiencia.

Para llevar a cabo la evaluación del impacto emocional y bajo el paradigma cualitativo, se le proporcionó al alumnado un cuestionario construido ad hoc y autoadministrado en el horario de clases, elaborado partiendo de las indicaciones de Bellocq y Gil Díaz (2010).

El cuestionario tenía el objetivo de obtener datos sobre su estado emocional a través de una serie de aspectos: cómo se sienten después de la sesión; lo que ha resultado fácil; lo que han aprendido; lo que ha sido difícil y lo que le gustaría hacer en sus futura aula relacionado con esta actividad (ver figura 2). 


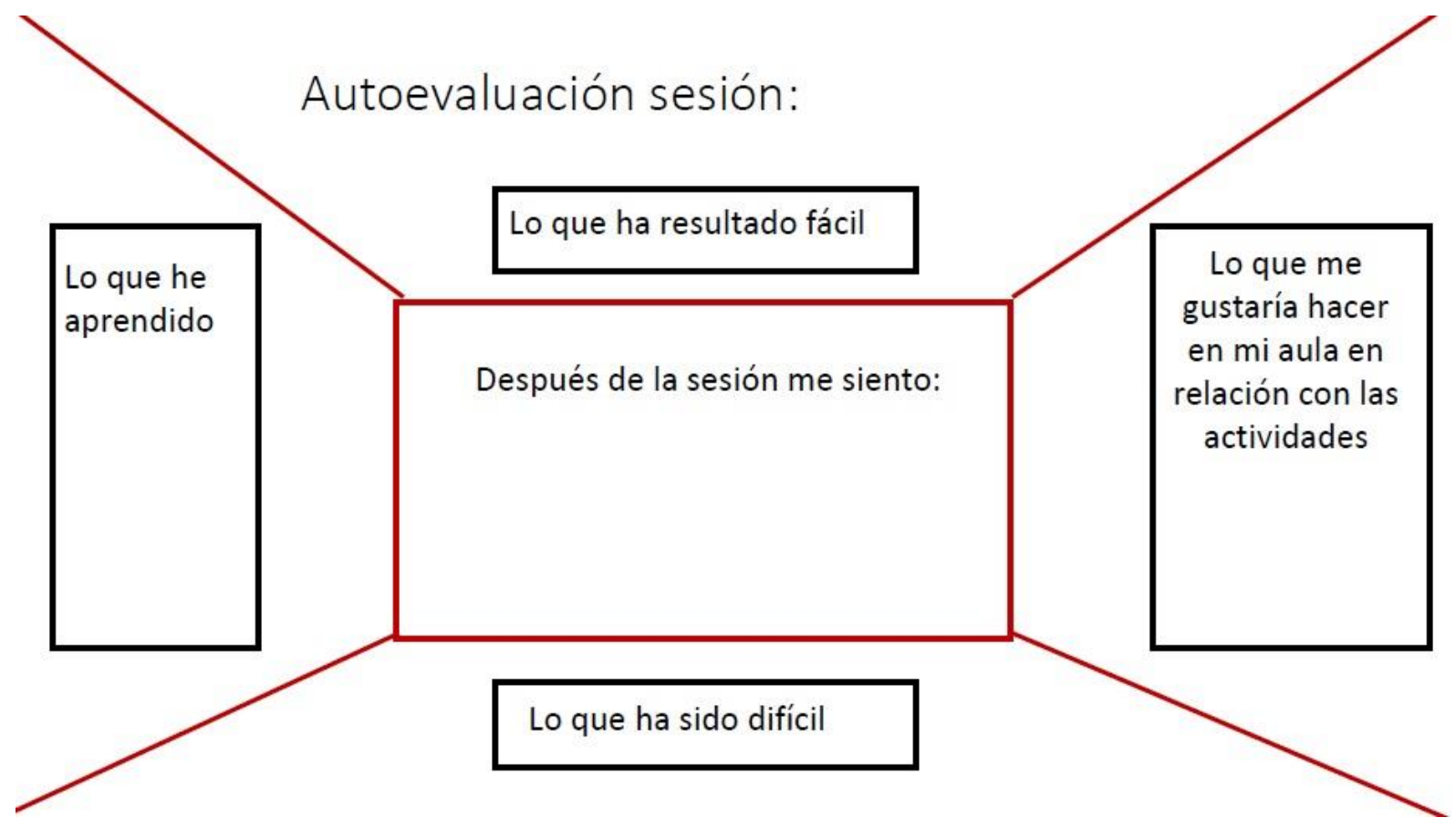

Figura 2. Cuestionario de autoevaluación emocional (Elaborada a partir de Bellocq y Gil Díaz, 2010)

La experiencia que aquí se describe tuvo lugar en la Facultad de Educación de la Universidad de Barcelona (UB) en la asignatura obligatoria "Educación Visual i Plástica a Primària", de 6 créditos, cursada en el primer cuatrimestre de segundo curso del Grado de Maestro de Educación Primaria. Cabe señalar, por tanto, que estamos hablando de una muestra de participantes muy homogénea, con un nivel muy semejante entre ellos, alumnos con un criterio basado en el deseo de formarse como maestros de primaria, aun con poca formación pero con el criterio necesario para desear crecer en cuanto a recursos emocionales.

La asignatura Educación Plástica y Visual, por su carácter de seminario se realiza logísticamente en grupos desdoblados, y se estructura en dos sesiones semanales de clase de dos horas cada una, a lo largo de dos semanas. El grupo de estudiantes habitual es de unos treinta alumnos y en total participaron en la experiencia 32 estudiantes ( 28 mujeres, 4 hombres), de edades comprendidas entre 20 y 24 años $(M=22.6 S D=0.5)$.

En cuanto al número de respuestas, al tratarse de un cuestionario autoadministrado con los asistentes, se ha obtenido un total de 22 respuestas, de las cuales 5 han tenido que ser desestimadas por estar incompletas o se inválidas al referirse a otros temas, en más de un $50 \%$, por lo que la muestra final se compone de 17 cuestionarios.

El cuestionario en papel se entregaba al alumnado al finalizar cada una de las sesiones con el objetivo de poder recoger de primera mano las impresiones, a la vez para aquellos alumnos que en el momento no disponían de tiempo o preferían entregarlo tras una pequeña reflexión cabía la posibilidad de entregarlo también a través del campus virtual. La aplicación de este cuestionario era voluntaria y anónima, y no interfería con la evaluación de los contenidos de la asignatura que son evaluados a través de una rúbrica específica. La intención de todo el proceso era comunicada a los estudiantes antes de iniciar las actividades y se informaba de que el objetivo que se pretendía guardaba relación con la mejora docente a través de la evaluación cualitativa y continua de los aprendizajes.

Esta investigación se enmarca dentro de los condicionantes del Informe Belmont (1978) y el Código de Buenas Prácticas en Investigación de la UB (2010) ${ }^{3}$. 


\section{Desarrollo de la propuesta}

La propuesta que aquí presentamos se compuso de siete actividades distribuidas a lo largo de dos sesiones, cinco en la primera y las dos restantes en la segunda.

\section{Primera sesión}

Se inició la propuesta con una breve introducción teórica sobre las distintas emociones y las reacciones tanto de origen neurofisiológico como comportamental o a nivel cognitivo que pueden comportar. En esta introducción se desarrolló en primer lugar el concepto de que el componente neurofisiológico coincide con el procesamiento emocional, que es un fenómeno inconsciente del propio cerebro que se activa a partir de una valoración automática. Es por este motivo que trabajando las emociones los futuros docentes pueden disminuir efectos de respuesta como la taquicardia, la sudoración, las secreciones hormonales o el cambio en los niveles de ciertos neurotransmisores. Por otro lado la propuesta también actúa sobre los componentes relacionados con el comportamiento, ya que en realidad tratan acerca de la manifestación externa de la emoción y que podemos ser conscientes de ello a través de la comunicación verbal y no verbal en los propios alumnos (con la expresión de la cara, el tono, el ritmo de la voz, el movimiento corporal...). Por último también se reflexiona sobre aquellos componentes más cognitivos, que permiten tomar conciencia de la reacción psicofisiológica y cognitiva que sucede en una emoción y etiquetándola en función del dominio del lenguaje.

Para visualizar en un ejemplo concreto y tomar conciencia de las emociones básicas, nos referimos a aquellas que compartimos la mayoría de las culturas, se eligió en primer lugar un fragmento de la película InsideOut (2015) en el que se observa cómo la personificación de las emociones básicas interviene directamente en las acciones de la protagonista. Por otro lado, también se prosiguió a la lectura cooperativa del álbum ilustrado El monstruo de los colores de la arteterapeuta Ana Llenas. En él podemos ver como el monstruo protagonista, no sabe qué le pasa, en realidad tiene un lío con las emociones y ahora le toca deshacer el embrollo. Se trata de una historia sencilla y divertida, que puede servir tanto para el alumnado de Infantil como para el primer ciclo de primaria que introduce al lector en el fascinante lenguaje de las emociones de una forma muy sencilla.

A partir de este punto, y dado que tanto la primera como la segunda propuesta tratan las emociones asociadas a colores, se introdujo la teoría de los colores ${ }^{4}$, con la que se trabajó el concepto con los futuros docentes a través de 5 rincones de colores que estaban distribuidos en distintos puntos del aula (alegría=amarillo, tristeza=azul, rabia=rojo, miedo=negro, calma=verde). En cada uno de ellos encontrábamos elementos (objetos diversos, lecturas, prendas...) del mismo color y el grupo clase compartía las emociones que le despertaba la isla de objetos cromáticos, tras esta pequeña experiencia se mantuvo un debate sobre la teoría.

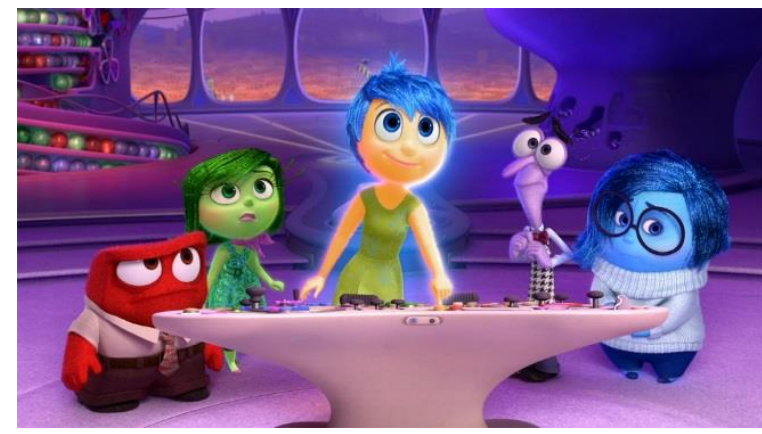

Imagen 1. Escena Inside Out..Pixar Animation Studios, Docter. P (2015)

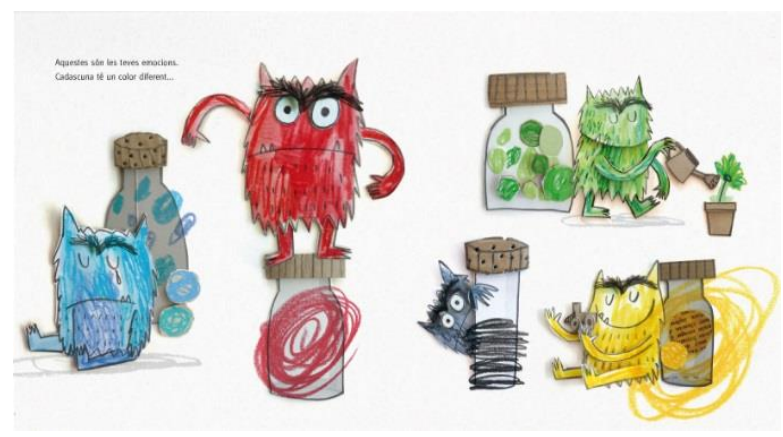

Imagen 2. Ilustración de El monstruo de los colores (Llenas, A., 2012) 
Una vez trabajadas las cinco emociones básicas en relación a los colores, la sesión continuó con la actividad "la red de emociones". Se dispuso de cinco ovillos de lana de distintos colores siguiendo la teoría trabajada previamente (rojo, azul, verde, negro, amarillo). En círculo, los alumnos debían pasarse los ovillos, de manera que el alumno que tiraba el ovillo al compañero relacionaba éste con un nombre o adjetivo que le sugiriera la emoción (por tanto estaban los cinco ovillos a la vez mezclándose). Al final, el visionado de una red de emociones entremezcladas refleja la metáfora de cómo en ocasiones podemos sentirnos, con una gran cantidad de emociones distintas que se cruzan entre ellas y que son difíciles de discernir para poder saber cómo tratarlas.

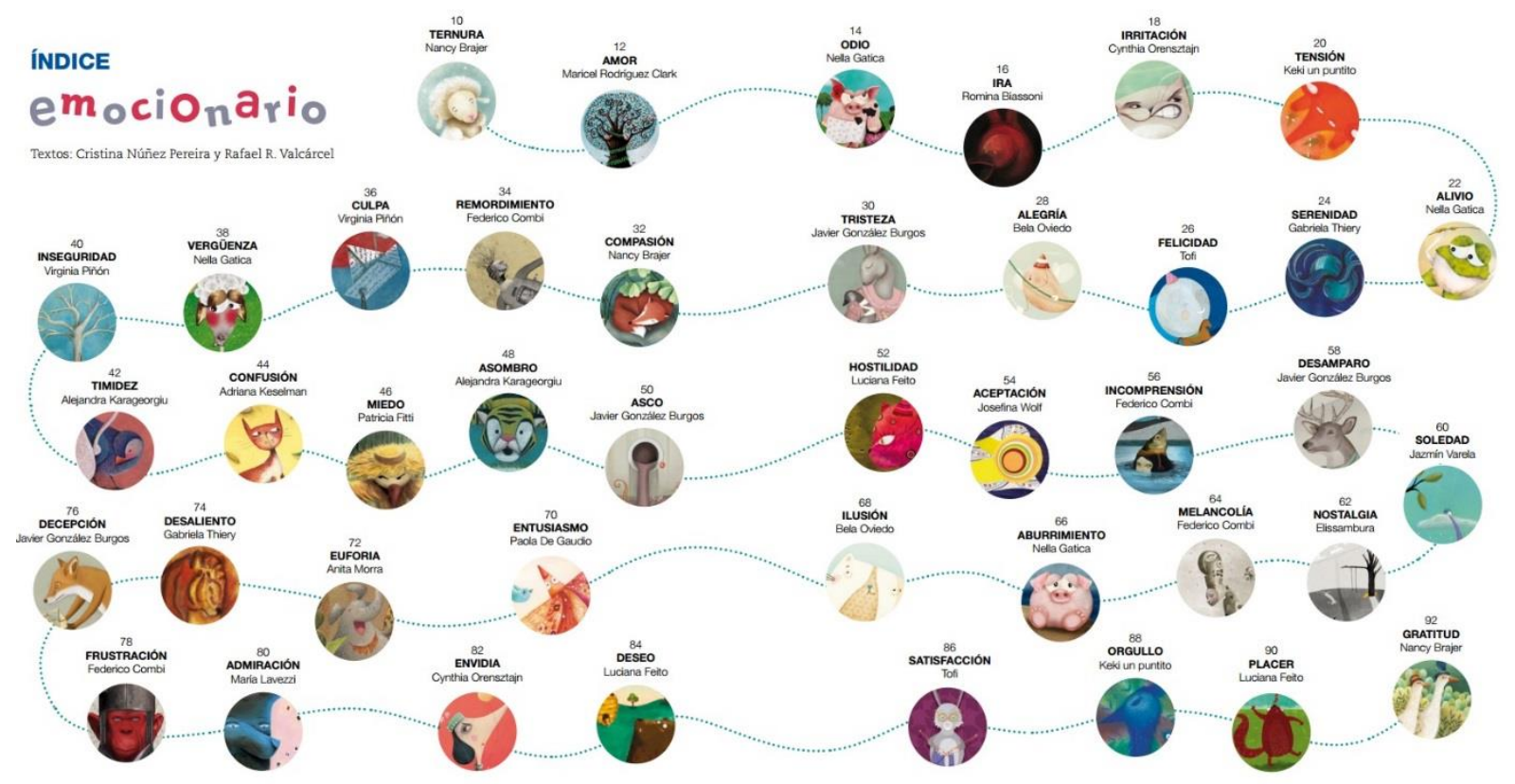

Imagen 3. Actividad "la red de emociones" (Fuente: los autores)

Para finalizar la sesión se trabajó con papel maché, que el alumnado había preparado previamente con el objetivo de montar un stop motion. Cada uno de los alumnos creó plásticamente un animal fantástico monocromo, que con la expresión de su rostro y su color debía representar una emoción. Por grupos de cinco, debían crear un pequeño film el guión del cual así como sus protagonistas debían hacer referencia a situaciones que pudieran desencadenar diversas emociones (representadas por los protagonistas). Recordemos que el planteamiento de las sesiones de Educación Visual y Plástica en primaria también contribuye al desarrollo de competencias básicas transversales relacionadas con la comunicación y el uso de la tecnología. De hecho las sesiones están planteadas de tal forma que están relacionadas entre sí y muchos de los procesos plásticos se concluyen con herramientas digitales.

\section{Segunda sesión}

La segunda sesión estuvo basada en una propuesta que pivota alrededor de un diccionario de emociones (Emocionario) que resulta elemental para trabajar con el alumnado los matices entre unas emociones y otras y así conseguir que los discentes adquieran un vocabulario tan amplio como su propia riqueza emocional. Para ello, se inició la sesión mostrando el mapa de las 42 emociones que presenta el libro Emocionario (Núñez y Valcárcel 2013). El aula estaba dotada de una alfombra a escala real creada a partir de la propuesta. La sesión empezó con cada uno de los alumnos situado en una emoción, en la casilla encontraban la definición dentro de un sobre, la compartían con sus compañeros y entre todos se buscaba una situación en la que se pudiera sentir este tipo de emoción. 


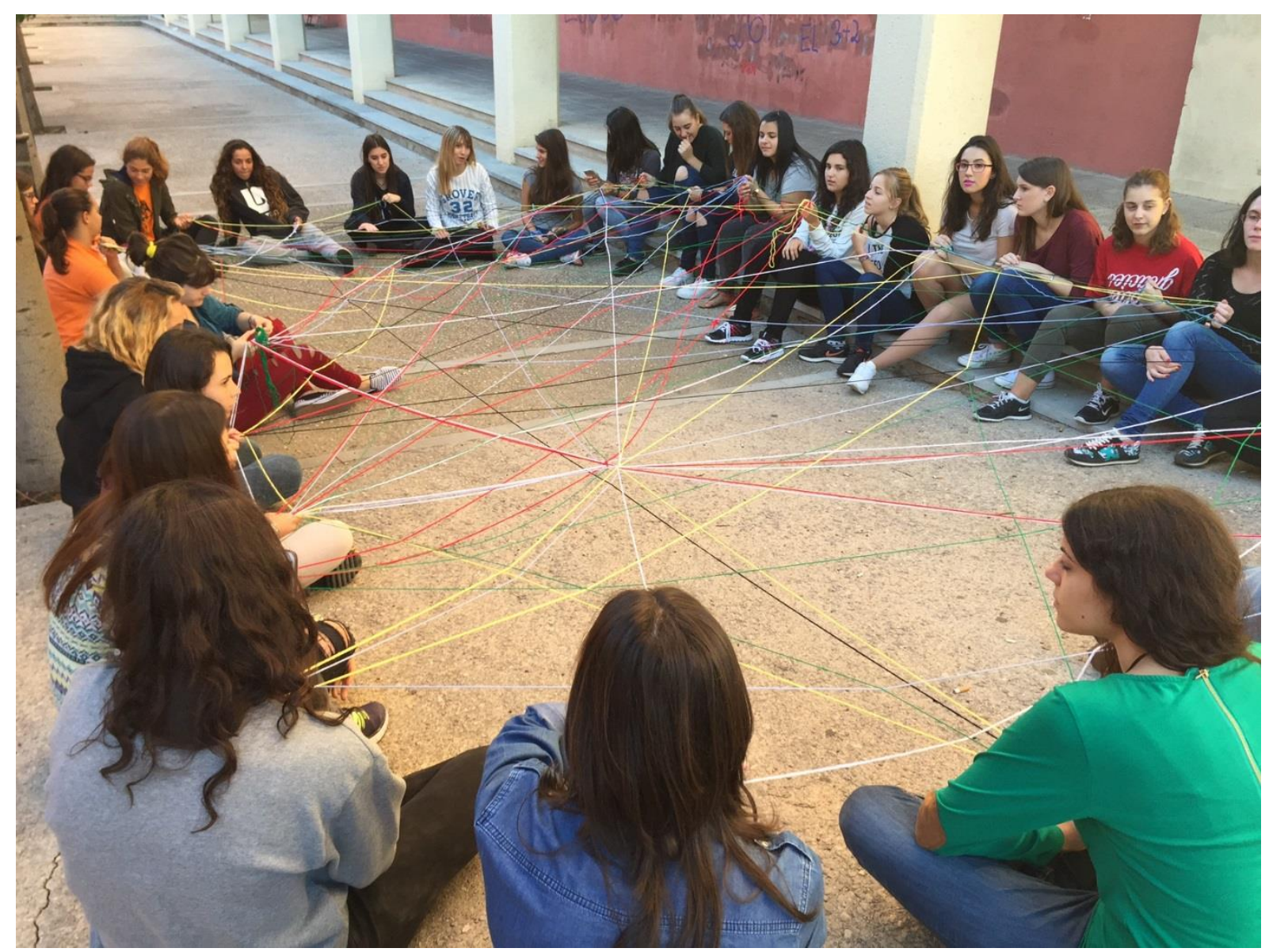

Imagen 4. Fotografía índice Emocionario (Nuñez y Valcárcel, 2013: 6-7)

Tras este rápido ejercicio, el alumnado encontró en su sitio un espejo un carboncillo y soporte para dibujar a mano alzada. Se inició la actividad "mírame a los ojos". El docente ayudado del contexto anterior en el que se había desarrollado cada emoción, escogía una batería de emociones que plantea a todo el alumnado. Cada alumno, a modo de trabajo individual mira en su espejo y trata de buscar la expresión que refleje dicha emoción. Tras verse en el espejo el alumno debía representar a modo de esbozo (sólo se disponía de 2 minutos por emoción) la expresión que había visto.

De la misma manera que en la sesión anterior, el alumnado concluyó el proceso con una propuesta relacionada con la imagen digital. La demanda consistía en una elaboración de un "emocionario facial". Los alumnos debían escoger 15 emociones, y fotografiar un primer plano de su cara de forma frontal y con el mismo encuadre en la que estuviesen expresando esas 15 emociones a modo de catálogo. Una vez tuviesen las 15 fotografías, se encuadernarían y se realizaría un corte a la altura de los ojos, boca y nariz, de tal forma que no fuese un libro al uso, sino que se pudiese configurar un hibrido entre las 15 emociones. 


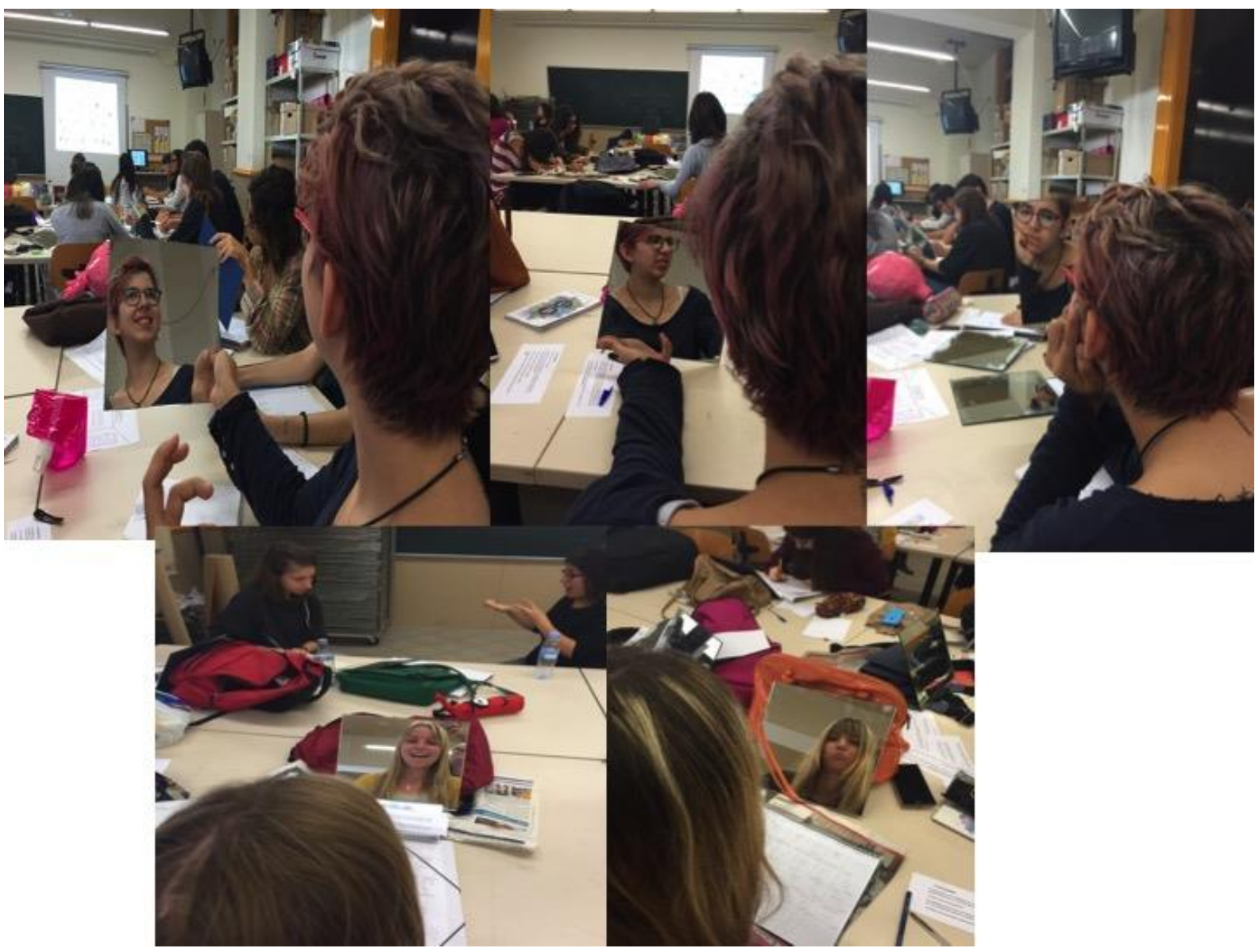

Imágenes 5. Actividad de guión. (Fuente: los autores)

A modo de resumen, la siguiente tabla recoge los principales detalles de las siete actividades distribuidas en las dos semanas.

\begin{tabular}{|c|c|c|c|c|c|}
\hline $\begin{array}{l}\text { Semana } \\
\text { (incluye } 2 \\
\text { sesiones) }\end{array}$ & Actividad & Título & $\begin{array}{l}\text { Competencia emocional } \\
5\end{array}$ & Objetivos & Materiales \\
\hline \multirow[t]{2}{*}{ Sesión 1a } & Act. 1 & $\begin{array}{l}\text { El mundo de las } \\
\text { emociones }\end{array}$ & $\begin{array}{l}\text {-Regulación emocional } \\
\text {-Competencia para la } \\
\text { vida y el bienestar } \\
\text { - Competencia social } \\
\text {-Conciencia emocional }\end{array}$ & $\begin{array}{l}\text {-Identificar cada una } \\
\text { de las emociones y } \\
\text { discernirlas } \\
\text {-Tomar conciencia } \\
\text { sobre la importancia } \\
\text { de las emociones } \\
\text { - Reconocer cómo las } \\
\text { emociones influyen en } \\
\text { nuestros estados } \\
\text { anímicos }\end{array}$ & $\begin{array}{l}\text { Film InsideOut } \\
\text { Álbum ilustrado El } \\
\text { mostre dels colors }\end{array}$ \\
\hline & Act. 2 & $\begin{array}{l}\text { La red de } \\
\text { emociones }\end{array}$ & $\begin{array}{l}\text {-Conciencia emocional } \\
\text {-Competencia social }\end{array}$ & $\begin{array}{l}\text {-Tomar conciencia } \\
\text { sobre la importancia } \\
\text { de las emociones }\end{array}$ & $\begin{array}{l}\text { Cinco ovillos de lana } \\
\text { con los colores } \\
\text { correspondientes a las } \\
\text { distintas emociones } \\
\text { alegría=amarillo, } \\
\text { tristeza=azul, } \\
\text { rabia=rojo, } \\
\text { miedo=negro, } \\
\text { calma=verde }\end{array}$ \\
\hline Sesión 1b & Act. 3 & Stop(E)motion & -Conciencia emocional & $\begin{array}{l}\text {-Crear un personaje } \\
\text { que exprese }\end{array}$ & $\begin{array}{l}\text { Cámaras fotográficas } \\
\text { Personajes para el } \\
\text { stopmotion }\end{array}$ \\
\hline
\end{tabular}




\begin{tabular}{|c|c|c|c|c|c|}
\hline & & & & $\begin{array}{l}\text { emociones atendiendo } \\
\text { al color y la expresión } \\
\text { - Proponer situaciones } \\
\text { que pudieran } \\
\text { desencadenar diversas } \\
\text { emociones }\end{array}$ & \\
\hline Sesión 2a & Act. 4 & $\begin{array}{l}\text { El recorrido de } \\
\text { las emociones }\end{array}$ & $\begin{array}{l}\text {-Conciencia emocional } \\
\text {-Competencia social }\end{array}$ & $\begin{array}{l}\text {-Reconocer las } \\
\text { diferentes emociones }\end{array}$ & $\begin{array}{l}\text { Álbum ilustrado } \\
\text { Emocionacio }\end{array}$ \\
\hline \multirow[t]{2}{*}{ Sesión 2b } & Act. 5 & $\begin{array}{l}\text { Mírame con mis } \\
\text { ojos }\end{array}$ & $\begin{array}{l}\text {-Regulación emocional } \\
\text {-Competencia social }\end{array}$ & $\begin{array}{l}\text {-Expresar emociones } \\
\text { utilizando la expresión } \\
\text { facial y el lenguaje } \\
\text { plástico }\end{array}$ & $\begin{array}{l}\text { Espejos } \\
\text { Cámaras fotográficas } \\
\text { Papel }\end{array}$ \\
\hline & Act. 6 & $\begin{array}{l}\text { Mi emocionario } \\
\text { facial }\end{array}$ & -Conciencia emocional & & Cámaras fotográficas \\
\hline
\end{tabular}

\section{Resultados}

A continuación presentamos los resultados partiendo del análisis crítico de los datos obtenidos:

Un amplio porcentaje del alumnado hace referencia a un mayor conocimiento de las propias emociones, constatable a través de manifestaciones tales como:

(3) aprendí a compartir conmigo misma mi expresión facial y a perder la vergüenza de verme reflejada;

(14) aprendí a conocerme un poco más

(10) me siento orgullosa e innovadora porque he podido ver una parte de mí no había descubierto anteriormente.

(9) Al mismo tiempo que contribuyó a la superación de inseguridades o la timidez: me siento más segura;

(8) me siento bien conmigo misma, a medida que pasaba el tiempo era más fácil y más divertido;

(5) me siento bien, ya que he conseguido perder la vergüenza;

(1) he aprendido a desinhibirme un poco.

También podemos afirmar que ha contribuido a que, en algunos casos, el alumnado reflexionase sobre la necesidad de formación en el ámbito de educación emocional, como se aprecia en los siguientes comentarios:

(18) hay muchas emociones que son muy similares en sí, pero que no son las mismas y muchas veces no somos consciente;

(21) me he dado cuenta de que no conocemos bien todas las emociones que tenemos y que deberíamos de trabajar más con ellas, así como a nuevos aprendizajes sobre el tema: he aprendido cosas nuevas (...) emociones que realmente no conocía;

(5) aprendí el significado de algunas emociones que no son muy corrientes en nuestro vocabulario.

Es destacable como el alumnado ha expresado como han podido reflexionar sobre el papel de las emociones en el arte, $\mathbf{y}$ de este como medio de expresión emocional, tal como se observa en declaraciones como:

(16) aprendí a plasmar términos abstractos como son las emociones en actividades artísticas;

(21) también aprendí que la expresión artística puede ser un medio para trabajar las emociones. 
En lo que respecta a la cuestión vinculada a la utilización de algunos de los recursos en sus futuras aulas, una amplia mayoría propone una adaptación de la actividad del espejo en función de la edad del alumnado.

Por otra parte, se pone de manifiesto que el alumnado es consciente de la importancia de la educación emocional y de la necesidad de trabajar habitualmente sobre ella y no en actividades o talleres aislados, tal como comenta:

(22) intentaría hacer habitualmente ejercicios en pequeñas sesiones de 10 minutos sobre emociones para que los alumnos se expresen y se sientan más cómodos en clase.

También se recogen otras aportaciones en las que se vincula la educación emocional con otras áreas del currículo a través de propuestas como:

(20) añadiría la posibilidad de hacer sonidos y frases relacionadas con las emociones o poniendo a los alumnos en determinadas situaciones para que las experimentase;

(16) trabajar las emociones con objetos de la naturaleza.

Así mismo, se han encontrado referencias a la dinámica de las sesiones, tales como:

(20) he aprendido cosas nuevas, tanto la dinámica como emociones que realmente no conocía;

(14) me parece muy interesante hacer clases tan divertidas, diferentes y útiles para los alumnos, Siento que con estas clases aprendí de verdad a darme cuenta de la línea educativa que me gustaría utilizar en mi futuro trabajo como maestra.

\section{Conclusiones}

Los resultados ponen de manifiesto un desconocimiento generalizado acerca de la educación emocional, tanto en el reconocimiento de las propias emociones como el de los recursos educativos para potenciarla en sus futuros contextos laborales. Resulta sorprendente e incluso preocupante el desconocimiento manifestado por los participantes, aunque es más habitual de lo que se podría suponer, dada la inexistencia de ninguna asignatura dedicada a esta temática en el grado de formación de maestros.

La educación emocional forma parte de contextos no formales así como de cursos de formación permanente y otros modelos como congresos, jornadas, etc. Que intentan llevar a los profesionales de la educación este tipo de formación transversal de forma interdisciplinar. Asimismo, se constata la necesidad de vincular las experiencias de educación emocional con ciertas dinámicas metodológicas docentes que permitan mayor flexibilidad en el aula

Así pues, atendiendo a los resultados obtenidos y las referencias extraídas de las manifestaciones de los participantes, podemos afirmar que la experiencia desarrollada ha contribuido a un mayor conocimiento de las propias emociones por parte del alumnado y, en algunos casos, a un incremento de seguridad y autoestima. Así mismo, ha generado una reflexión sobre el papel y la importancia de las emociones en el contexto educativo y, por ende, a la necesidad de formación en dicho ámbito.

Por otro lado, hemos podido constatar que la batería de actividades realizada ha servido para dotar a los futuros docentes de herramientas para implementar en sus respetivas aulas, lo cual se muestra a partir de las propuestas de adaptación que realizarían. Podemos afirmar que, casi la totalidad de los participantes son sensibles a la importancia de la educación emocional, y conscientes de la necesidad de trabajar habitualmente con ella de manera global y transversal a todas las materias,

En lo que respecta al área de expresión artística, es posible observar que parte del alumnado ha reflexionado sobre las artes plásticas como medio de expresión emocional. 
A tenor del desarrollo de la experiencia y apoyándonos en los datos obtenidos, podemos afirmar que ésta ha sido un éxito, en tanto que ha dotado al alumnado de nuevas competencias instrumentales partiendo del desarrollo de sus propias competencias emocionales.

En definitiva, el vertiginoso ritmo al que se producen los cambios en el contexto del siglo XXI ya no nos hacen solo pensar en qué enseñamos, sino que nos invita a reflexionar también en el cómo, el por qué y en el para qué lo hacemos. Este nuevo paradigma genera grandes incertidumbres y fuertes ambigüedades, por lo que creemos que entre los retos a los que se enfrenta el sistema educativo actual se encuentra el dotar al profesorado de las herramientas necesarias para poder hacer frente a la modernidad de nuestros días de manera que puedan responder a la tarea de, a través del ejercicio de su labor docente, contribuir a que el discente desarrolle las competencias necesarias para una vida competente y saludable.

\section{Notas}

${ }^{1}$ Para seleccionar las Universidades se siguió el Academic Ranking of World Universities 2016 results THE Student (ARWU). Las seleccionadas fueron la Universidad de Barcelona, Universidad Autónoma de Barcelona, Universidad Autónoma de Madrid, Universidad Complutense de Madrid, Universidad de Granada, Universidad de Valencia, Universidad de Santiago de Compostela, Universidad de Sevilla, Universidad del País Vasco, Universidad de Zaragoza, Universidad de Huelva, Universidad de Valladolid, Universidad de Oviedo, Universidad Rovira i Virgili y Universidad de Salamanca.

${ }^{2}$ Antes denominadas "competencias básicas" (LOE, Ley Orgánica 2/2006, de 3 de mayo).

${ }^{3}$ Este código recoge los siguientes conceptos: confidencialidad, beneficencia, justicia, riesgos, mínima intervención y libre consentimiento informado.

${ }^{4}$ Siguiendo la idea de Heller (2004) entendemos que la asociación que se establece entre los colores y los sentimientos no son accidentales, sino experiencias enraizadas en nuestro lenguaje y pensamiento desde nuestra infancia. Ahora bien, dado que existen más sentimientos que colores, debemos aceptar la ambigüedad e incluso la contradicción de sus efectos. Así mismo, generalmente estos no aparecen aislados, sino que el contexto y la conexión con otros colores determinará cómo es percibido y su efecto.

${ }^{5}$ Bisquerra y Pérez Escoda (2007) distinguen las siguientes competencias emocionales: consciencia emocional; regulación emocional; autonomía emocional; competencia social; competencia para la vida y el benestar

\section{Bibliografía}

Bauman, Z. (2005). Los retos de la educación en la modernidad líquida. Barcelona: Gedisa.

Bellocq, G. \& Gil Díaz, M. J. (2010). Tocar el Arte. Madrid: Kaleida forma.

Betes, M. (Coord.) (2000). Fundamentos de musicoterapia. Madrid: Morata.

Bisquerra, R. (2000). Educación emocional y bienestar. Barcelona: Praxis.

Bisquerra, R. \& Pérez, N. (2007). Las competencias emocionales. Educación XXI, 10, 61-82. doi: http://dx.doi.org/10.5944/educxx1.1.10.297

Burset, S. Calderón, D. \& Gustems, J. (2016). Proyectos artísticos interdisciplinares. La creación al servicio del bienestar. Barcelona: Edicions Universitat de Barcelona.

Cabello, R. Ruiz-Aranda, D. \& Fernández-Berrocal, P. (2010). Docentes emocionalmente inteligentes. Revista Electrónica Interuniversitaria de Formación del Profesorado, 13(1), 41-49. 
Callejas, C. Santamaría, A. P. \& Albornoz, P. (2014). El arteterapia como herramienta para mejorar la autoestima en adultos con discapacidad física y mental. Quito: Universidad San Francisco de Quito.

Callejón, M. D. \& Granados, I. M. (2003). Creatividad, expresión y arte: Terapia para una educación del siglo XXI. Un recurso para la integración. Escuela abierta: revista de Investigación Educativa, 6, 129-148.

Cejudo, J. López-Delgado, M. \& Rubio, M. J. (2015). La formación en educación emocional de los docesntes: una visión de los futuros maestros. Revista Española de Orientación y Psicopedagogía, 26 (3), 45-62. doi: http://dx.doi.org/10.5944/reop.vol.26.num.3.2015.16400

Cornejo, S. \& Brik, L. (2003). La representación de las Emociones en la Dramaterapia. Argentina: Panamericana

Delors, J. (Coord.) (1996). La Educación encierra un tesoro. Informe a la UNESCO de la Comisión Internacional sobre la Educación para el siglo XXI. Madrid: Santillana-Unesco.

Duncan, N. (2007). Trabajar con las Emociones en Arteterapia. Arteterapia. Papeles de arteterapia y educación artística para la inclusión social, 2, 39-49. doi: http://dx.doi.org/10.5209/rev ARTE.2007.v2.9757

Fernández-Abascal, E. G. Chóliz, M. Palermo, F. \& Martínez, F. (1997). Cuaderno de prácticas de motivación y emoción. Madrid: Ediciones Pirámide.

Fernández-Berrocal, P. \& Ruiz-Aranda, D. (2008). La inteligencia emocional en la Educación. Revista Electrónica de Investigación Psicoeducativa, 6(2), 421-436.

Goleman, D. (1995). Emotional intelligence: Why it can matter more than IQ for character, health and lifelong achievement. Nueva York: Bantam Books.

Goleman, D. (1999). La práctica de la inteligencia emocional. Barcelona: Kairós.

Gustems, J. \& Sánchez, L. (2015). Aportaciones de la Psicología Positiva aplicadas a la formación del profesorado. Estudios sobre Educación, 29, 9-28. doi: http://dx.doi.org/10.15581/004.29.9-28

Heller, E (2004). Psicología del color. Cómo actúan los colores sobre los sentimientos y la razón. Barcelona . Editorial Gustavo Gili, S.L.

Informe Belmont. (1978). National Comission for the Protection of Human Subjects of Biomedical and Behavioral Research. The Belmont Report, Ethical Principle and Guidelines for the protection of human research subjects. Washington: DHEW.

Izard, C. E. (2001). Emotional intelligence or adaptative emotions. Emotion, 1(3), 249-257.

Izard, C. E. (2007). Basic emotions, natural kinds, emotion schemas, and a new paradigm. Perspectives on psychological science, 2(3), 260-280. doi: http://dx.doi.org/10.1111/j.1745-6916.2007.00044.x

Klein, J. P. (2006). Arteterapia: una introducción. Barcelona: Octaedro.

Llenas, A. (2012). El monstre de colors. Barcelona: Flamboyant

LOE, Ley Orgánica 2/2006, de 3 de mayo «BOE» núm. 106, de 4 de mayo de 2006, páginas 17158 a 17207.

LOMCE, Ley Orgánica 8/2013, de 9 de diciembre «BOE» núm. 295, de 10 de diciembre de 2013, páginas 97858 a 97921.

Marina, J. A., Pallicer, C. \& Manso, J. (2015) Libro blanco de la profesión docente y su entorno escolar. Madrdid: Publicaciones MECD.

Mathews, A. \& MacLeod, C. (1994). Cognitive approaches to emotion and emotional disorders. Annual review of psychology, 45, 25-47. doi: http://dx.doi.org/10.1146/annurev.ps.45.020194.000325

Mayer, J. D. \& Salovey, P. (1993). The intelligence of emotional intelligence. Intelligence, 17(4), 433-442. 
Molero, C. Saiz, E. \& Esteban, C. (1998). Revisión histórica del concepto de inteligencia: una aproximación a la inteligencia emocional. Revista Latinoamericana de Psicología, 30(1), 11-30 Fundación Universitaria Konrad Lorenz Bogotá, Colombia.

Nuñez, C. y Valcárcel, R. (2013). Emocionario. Madrid: Palabras aladas.

Orden ECD/65/2015, de 21 de enero «BOE» núm. 25, de 29 de enero de 2015, páginas 6986 a 7003.

Oriola, S. \& Gustems, J. (2015). Música y adolescencia: usos, funciones y consideraciones educativas. Universitas Tarraconensis, Revista de Ciències de l'Educació, 2015(2), 27-42. doi: http://dx.doi.org/10.17345/ute.2015.1.660

Palmero, F. (1996). Aproximación biológica al estudio de la emoción. Anales de psicología, 12(1), 61-86.

Palomera, R. Fernández-Berrocal, P. \& Brackett, M.A (2008). La inteligencia emocional como una competencia básica en la formación inicial de los docentes: algunas evidencias. Revista Electrónica de Investigación Psicoeducativa, 15, 6(2), 437-454.

Pixar Animation Studios, Docter, P (2015). Inside Out [cinta cinematográfica]. Estados Unidos: Walt Disney Pictures

Riera, R. (2010). La connexió emocional. Barcelona: Octaedro.

Rodríguez, E. (2007). Aplicaciones del Arteterapia en aula como medio de prevención para el desarrollo de la autoestima y el fomento de las relaciones sociales positivas: "me siento vivo y convivo". Arteterapia: papeles de arteterapia y educación artística para la inclusión social, 2, 275-292. doi: http://dx.doi.org/10.5209/rev ARTE.2007.v2.9842

Russell, J. A. (1980). A circumplex model of affect. Journal of Personality and Social Psychology, 39, 11611178.

Souza, M. C. (2011). Pesquisa social: teoria, método e criatividade. Brasilia: Vozes Ltda.

Sternberg, R. J. (1985). Beyond IQ: A triarchic theory of human intelligence. Cambridge: CUP Archive.

Sutton, R.E. \& Wheatly, K.F. (2003). Teacher's Emotions and Teaching: A review of the literatura and directions for future research. Educational Psychology Review, 15(4), 327-358. doi: http://dx.doi.org/ 10.1023/A:1026131715856

Universidad de Barcelona. (2010). Código de Buenas Prácticas en Investigación. Barcelona: Universidad de Barcelona.

Zaccagnini, J. L. (2008). La comprensión de la emoción: una perspectiva psicológica. En A. A. Mesas (Ed.), Educación emocional y convivencia en el aula (pp. 31-84). Madrid: Ministerio de Educación. 